Military Technical College, Kobry El-Kobbah, Cairo, Egypt

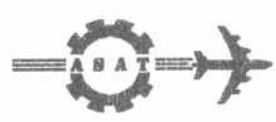

$9^{\text {th }}$ International Conference

On Aerospace Sciences \&

Aviation Technology

\title{
ON SIMULATING ACOUSTIC-SHOCK WAVES INTERACTION
}

KHALID M. HOSNY *, ISMAIL A. ISMAIL“" and AWATEF A. HAMED"-

\section{ABSTRACT}

Numerical simulation of acoustic-shock waves interaction in quasi-one dimensional convergent-divergent nozzles is performed. The time dependent nature of flow field is initiated by using very small amplitude acoustic wave that incident on the inlet of the nozzle. Computations are performed using 3-point fourth-order compact implicit McCormick-type scheme to approximate the spatial derivatives and the two steps 5-6 alternating 2-N storage Low-Dispersion and -Dissipation Runge-Kutta (LDDRK) method as a time marching scheme. Artificial damping terms were used in damping non-physical very high wave numbers waves. The obtained numerical solutions with acoustic perturbation are presented.

Key Words: Aeroacoustics, compact schemes, Runge-Kutta, Fourier transform.

* Lecturer, Dept. of Computer Science, College of Computers \& Informatics, Zagazig University, Zagazig, Egypt.

** Professor. Dept. of .Computer Science and Dean of College of Computers \& Informatics, Zagazig University, Zagazig, Egypt.

*** Professor, Dept. of Aerospace Engineering \& Engineering Mechanics, College of Engineering, University of Cincinnati, Cincinnati, Ohio, USA. 


\section{INTRODUCTION}

Numerical simulations of acoustic waves that interact with shock waves encounter a number of challenges. The first and the obvious one is the large disparity between the pressure fluctuations that represent the acoustic and the mean flow where the considered acoustic wave is specified to be in the order $10^{-6}$ times the dynamic pressure. The second obvious challenging is the shock waves. The interaction between acoustic and shock waves leads to more complications in the solution procedures where each member of this interaction process need special attention and treatment. Resolving shock wave requires dissipation to suppress the undesired waves and wiggles formed in the thin region around shock; while, the acoustic wave required a careful treatment because its sensitive nature. Therefore, the numerical methods used to solve this problem must simultaneously capture the shock and accurately resolve the very high frequency acoustic wave. In addition, these numerical methods need to be simple and efficient for long time integration so that it can be used in simulating flows with complex configurations.

Finite-difference schemes are classified mainly into two classes, explicit and compact schemes. Explicit schemes use large number of grid nodes for high accuracy discretization while the compact schemes employ smaller stencils. In addition, compact schemes are more accurate than the equivalent explicit ones. The main disadvantage of the compact schemes is the matrix inversion at each grid point, which means higher cost of computational time and storage. In the past, many researchers used the compact difference schemes in solving various flow problems (e.g. Refs. [1-3]). Recently, Hixon and Turkel [4] derived a new compact implicit McCormick-type difference scheme. This scheme is fourth-order accurate. Hu et al [5] derived an optimized version of Runge-Kutta time marching schemes called LowDispersion and -Dissipation Runge-Kutta LDDRK schemes. Both single-step and two-steps alternating schemes are included. The derivation of these schemes based on the Dispersion-Relation-Preserving methodology that minimizes the dissipation and dispersion errors for wave propagation. The optimized LDDRK schemes are fourth-order accurate in time for linear problems while the accuracy reduced to be a second-order for nonlinear problems. The implementation of LDDRK requires $3-N$ storage, where $N$ is the number of degrees of freedom of the system (i.e., number of grid points multiplied by number of variables). Recently, Stanescu and Habashi [6] presented $2-\mathrm{N}$ storage formulation for LDDRK schemes. This formulation provides fourth-order accuracy in time for both linear and non-linear wave propagation problems.

According to memory requirements for physical problems with complex configurations, it is desirable to use $2-N$ storage LDDRK schemes. In the present paper, we combine the fourth-order compact MacCormack-type scheme with the 2-N storage LDDRK schemes. To assess the numerical stability of the considered scheme we use Fourier analysis for the fully discretized equations. In order to suppress the undesired high frequency spurious waves that are generated through the numerical solution we add explicit numerical damping terms. The additional damping terms are designed by using the damping method developed by Tam et al [7]. Through the next sections we present the numerical solution for very small amplitude acoustic wave that incident on the inlet of quasi-one dimensional 
convergent-divergent nozzles with two different cases. First, no shock is considered while in the second a normal shock in the divergent part of the nozzle is considered.

\section{SPATIAL DISCRETIZATION}

For a computational domain consists of grid points from $i=0$ to $i=i \max$, the approximation of $D_{j}$, which represent the spatial derivative of the function $f$ at interior grid points of the computational domain is obtained from the equations:

$$
\begin{gathered}
D_{j}^{B}=\frac{1}{4 \Delta x}\left\{-5 f_{j-1}+4 f_{j}+f_{j+1}\right\}-\frac{1}{2} D_{j-1}^{B} \\
D_{j}^{F}=\frac{1}{4 \Delta x}\left\{-f_{j-1}-4 f_{j}+5 f_{j+1}\right\}-\frac{1}{2} D_{j+1}^{F} \\
D_{j}=\frac{D_{j}^{F}+D_{j}^{B}}{2}
\end{gathered}
$$

The superscripts $F$ and $B$ refer to forward and backward difference respectively.

\subsection{Boundary Stencils}

One-sided derivatives are used at the boundary points. For the boundary point approximation, Hixon has derived the following forms for both forward and backward sweeps for the sixth-order compact scheme.

Left boundary point $(j=0)$ :

$$
\begin{aligned}
& D_{0}^{F}=S_{0} f_{0}+S_{1} f_{1}+S_{2} f_{2}+S_{3} f_{3}+S_{4} f_{4} \\
& D_{0}^{B}=E_{0} f_{0}+E_{1} f_{1}+E_{2} f_{2}+E_{3} f_{3}+E_{4} f_{4}
\end{aligned}
$$

Right boundary point $(j=i \max )$ :

$$
\begin{aligned}
& D_{i \max }^{F}=-E_{0} f_{i \max }-E_{1} f_{i \max -1}-E_{2} f_{i \max -2}-E_{3} f_{i \max -3}-E_{4} f_{i \max -4} \\
& D_{i \max }^{B}=-S_{0} f_{i \max }-S_{1} f_{i \max -1}-S_{2} f_{i \max -2}-S_{3} f_{i \max -3}-S_{4} f_{i \max -4}
\end{aligned}
$$

The coefficients of boundary stencils are:

$$
\begin{aligned}
& S_{0}=-19 / 9, S_{1}=37 / 9, S_{2}=-19 / 6, S_{3}=13 / 9 \text { and } S_{4}=-5 / 18 \\
& E_{0}=-37 / 18, E_{1}=35 / 9, E_{2}=-17 / 6, E_{3}=11 / 9 \text { and } E_{4}=-2 / 9
\end{aligned}
$$

\section{TIME MARCHING SCHEME}

The considered time marching scheme is the optimized 5-6, $2 \mathrm{~N}$-storage (LDDRK) [6]. This scheme is two-steps alternating scheme, in the first step we use five stages and in the second we use six stages. The scheme is fourth-order accurate in time for 
linear and nonlinear problems. To describe this scheme let us assume the model equation $\partial U / \partial t=F(U)$, where $U$ represents the vector of conservative variables. Starting with an initial solution $U^{(n-1)}$, we perform the following two steps:

\section{1- Step with five stages.}

$$
\begin{aligned}
& K_{i}=F\left(U^{(n-1)}+\Delta t \sum_{j=1}^{i-1} c_{i j} K_{j}\right) \\
& U^{(n)}=U^{(n-1)}+\sum_{j=1}^{5} b_{5}, K_{i}
\end{aligned}
$$

With the coefficients values:

$$
\begin{aligned}
& c_{21}=0.2687454 \\
& c_{32}=0.8014706 \\
& c_{31}=-0.216242573 \\
& c_{43}=0.5051570 \\
& c_{42}=-0.230947251 \\
& c_{41}=0.408496801
\end{aligned}
$$$$
c_{54}=0.5623568
$$$$
c_{53}=0.088619374
$$$$
c_{52}=0.620354186
$$$$
c_{51}=-0.106644938
$$

$$
\begin{aligned}
& b_{51}=0.132438181 \\
& b_{52}=0.225255541 \\
& b_{53}=0.281939207 \\
& b_{54}=0.301360635 \\
& b_{55}=0.0590065
\end{aligned}
$$

\section{2- Step with six stages.}

$$
\begin{aligned}
& K_{i}=F\left(U^{(n-1)}+\Delta t \sum_{j=1}^{i-1} d_{, j} K_{j}\right) \\
& U^{(n)}=U^{(n-1)}+\sum_{i=1}^{6} b_{6} K_{,}
\end{aligned}
$$

With the coefficients values:

$$
\begin{array}{llll}
d_{21}=0.1158488 & & d_{65}=1.0312849 & b_{66}=0.77698851 \\
d_{32}=0.3728769 & d_{54}=0.5798110 & d_{64}=-2.305651238 & b_{62}=-1.498253149 \\
d_{31}=-0.048691969 & d_{53}=-0.251410958 & d_{63}=4.67221773 & b_{01}=1.742235949 \\
d_{43}=0.7379536 & d_{52}=0.642887744 & d_{62}=-4.645000842 & b_{64}=-0.588553479 \\
d_{42}=-0.419671983 & d_{51}=-0.167840653 & d_{61}=2.165565508 & b_{64}=0.417581845 \\
d_{41}=0.301039008 & & & b_{64}=0.15
\end{array}
$$

\section{FOURIER ANALYSIS}

Fourier analysis provides an effective way to quantify the numerical errors (dispersion and dissipation errors) and resolution characteristics of the difference approximations. The background for Fourier analysis on discretized equations can be found in [8]. To assess the characteristics (dispersion, dissipation and stability) of 
the considered numerical methods using Fourier analysis, we must define the amplification factor. This factor is defined by the ratio of the numerical solution at time levels $n+1$ and $n$ in the wave number domain. For the time evolution equation $\partial U / \partial t=F(U)$, we define the amplification factor as $G=\widetilde{U}_{k}^{n+1} / \widetilde{U}_{k}^{n}$ where $\widetilde{U}_{k}^{n}$ is the spatial Fourier Transform of $U^{n}$. The amplification factor for the two steps alternating time advancing scheme is defined as $G=G_{1} G_{2} / G_{e}^{2}$ where $G_{1}$ is the amplification factor for the first step, $G_{2}$ is the amplification factor for the second step and $G_{e}$ is the exact amplification factor:

$$
G_{1}=1+\sum_{j=1}^{5} \alpha_{j}(-j \sigma)^{j}, G_{2}=1+\sum_{j=1}^{6} \beta_{j}(-j \sigma)^{j} \text { and } G_{e}=e^{(-j \sigma)}
$$

With the coefficient values: $\alpha_{1}=1, \alpha_{2}=1 / 2, \alpha_{3}=1 / 6, \alpha_{4}=1 / 24, \alpha_{5}=0.0036105$ and $\beta_{1}=1, \beta_{2}=1 / 2, \beta_{3}=1 / 6, \beta_{4}=1 / 24, \beta_{5}=0.0121101, \beta_{6}=0.00285919 . \quad j=\sqrt{-1}$, $\sigma=c k^{*} \Delta t, \Delta t$ is time step and $k^{*}$ is numerical wave number. According to the numerical wave number for the spatial scheme [9] with CFL number, we can write:

$$
\sigma=(C F L) \frac{0.5 \sin (2 k \Delta x)+8 \sin (k \Delta x)}{5+4 \cos (k \Delta x)}
$$

By writing the amplification factor $G$ as:

$$
G=|G| e^{-j \delta}
$$

where $|G|$ is the numerical dissipation. It must be noted that, the numerical scheme is stable when $|G|$ less than unity. In figure (1), we plot the numerical dissipation of the considered scheme for three different values of Courant-Friedrichs-Lewy (CFL) number. These values are selected as $1.16,1.183$ and 1.2 . The scheme is stable only when the numerical dissipation less than unity. Therefore, the stability limit of the considered scheme is $(C F L) \leq 1.183$.

\section{ARTIFICIAL DAMPING TERMS}

The explicit damping terms are added to the difference equations where the value of these terms controlled by a free parameter called the damping coefficient. The values of the 3-Point Tam's damping weight coefficients are:

$$
d_{0}=0.5 \text { and } d_{1}=d_{-1}=-0.25 \text {. }
$$

\section{CASE STUDY PROBLEM}

The considered problem is selected from the workshop on benchmark problem [10]. In this problem, the amplitude of the incident wave is in the order of $10^{-6}$ times the 
dynamic pressure that is based on the speed of sound of the incoming wave. The cross sectional area of the nozzle is plotted in figure (2) and defines by:

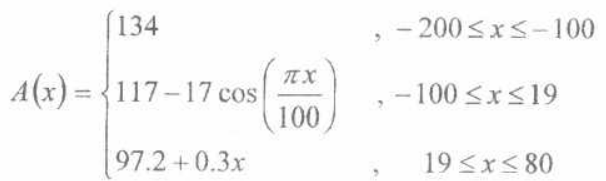

Upstream the nozzle $(x \leq-200)$, the incoming acoustic wave is specified by

$$
\left[\begin{array}{l}
u \\
p \\
\rho
\end{array}\right]=\left[\begin{array}{l}
M \\
\frac{1}{\gamma} \\
1
\end{array}\right]+\left[\begin{array}{l}
1 \\
1 \\
1
\end{array}\right] \varepsilon \sin \left[\omega\left(\frac{x}{1+M}-t\right)\right]
$$

Where $M=0.5$ is the Mach number, $\varepsilon=10^{-6}$ is the amplitude of the acoustic wave and $\omega=0.1 \pi$ is the angular frequency of the acoustic wave. The governing equations are the quasi-one dimensional time dependent Euler equations:

$$
\frac{\partial(A U)}{\partial t}+\frac{\partial(A F)}{\partial x}=H
$$

Where

$$
U=\left[\begin{array}{c}
\rho \\
\rho u \\
\rho E
\end{array}\right], F=\left[\begin{array}{c}
\rho u \\
\rho u^{2}+p \\
(\rho E+p) u
\end{array}\right], H=\left[\begin{array}{c}
0 \\
p d A / d x \\
0
\end{array}\right]
$$

The equation of state is

$$
p=(\gamma-1) \rho\left(E-0.5 u^{2}\right)
$$

Where $\rho, u, p$ and $E$ are density, velocity, pressure and the total specific energy respectively. $\gamma=C_{p} / C_{p}$. It has the constant value 1.4 . Since the nozzle cross sectional area $A(x)$ is independent of time, and for the governing equations to be easier to use with the time advancing numerical schemes we do some algebraic manipulations and then write the governing equations in the conservative form as follows:

$$
\begin{gathered}
\frac{\partial \rho}{\partial t}+\frac{\partial(\rho u)}{\partial x}+\frac{(\rho u)}{A} \frac{d A}{d x}=0 \\
\frac{\partial(\rho u)}{\partial t}+\frac{\partial}{\partial x}\left(\frac{3-\gamma}{2} \frac{(\rho u)^{2}}{\rho}+(\gamma-1)(\rho E)\right)+\frac{(\rho u)^{2}}{\rho A} \frac{d A}{d x}=0
\end{gathered}
$$




$$
\begin{aligned}
\frac{\partial(\rho E)}{\partial t}+\frac{\partial}{\partial x}(\gamma & \left.\frac{(\rho E)(\rho u)}{\rho}-\frac{(\gamma-1)}{2} \frac{(\rho u)^{3}}{\rho^{2}}\right) \\
& +\frac{1}{A}\left(\gamma \frac{(\rho E)(\rho u)}{\rho}-\frac{(\gamma-1)}{2} \frac{(\rho u)^{3}}{\rho^{2}}\right) \frac{d A}{d x}=0
\end{aligned}
$$

To establish the normal shock in the nozzle, the exit pressure to inlet total pressure is specified to be 0.76 . This case with the shock is similar to the previous study done Bui and Mankbadi [11] using an unstructured finite volume algorithm. Initial conditions are computed by integrating the steady state governing equations analytically [10]. We compute and show the numerical values for the initial steady state variables in the two different cases (without and with shock) in figure (3) and figure (4) respectively. Mach numbers in the indicated two different cases are shown in figure (5) and figure (6) respectively.

\section{BOUNDARY CONDITIONS TREATMENT}

For the considered problem, the boundary conditions are divided into inflow and outflow boundary conditions. Inflow boundary conditions must allow the incoming acoustic waves to propagate into the computational domain. Also, the inflow boundary conditions must permit the reflected waves to leave the computational domain. The outflow boundary condition must allow the outgoing acoustic waves to pass without inducing non-physical reflections to the computational domain.

\subsection{Inflow Boundary Conditions}

At the inlet of the considered nozzle, we apply the radiation boundary condition of Tam and Webb [12]. Radiation boundary conditions will allow the incoming acoustic wave to propagate into the computational domain and at the same time permit the reflected waves to leave the computational domain without reflections. The radiation boundary conditions are derived from the asymptotic solutions of the governing equations. For the current problem, the following equations represent nonhomogeneous radiation boundary conditions that can be applied at the left boundary. The equations are:

$$
\frac{\partial}{\partial t}\left(\begin{array}{l}
\rho \\
u \\
p
\end{array}\right)=(1-M) \frac{\partial}{\partial x}\left(\begin{array}{l}
\rho \\
u \\
p
\end{array}\right)-\left(\begin{array}{l}
1 \\
1 \\
1
\end{array}\right) \frac{2 \omega \varepsilon}{(1+M)} \cos \left[\omega\left(\frac{x}{1+M}-t\right)\right]
$$

\subsection{Outflow Boundary Conditions}

At the exit of the nozzle (outflow boundary) we apply Thompson non-reflecting boundary condition [12]. These boundary conditions based on the theory of characteristics. The compatibility equations of the original governing equation (15) can be written in the following form:

$$
\frac{\partial p}{\partial t}-\rho c \frac{\partial u}{\partial t}+(u-c)\left(\frac{\partial p}{\partial x}-\rho c \frac{\partial u}{\partial x}\right)+\frac{\rho u c^{2}}{A} \frac{\partial A}{\partial x}=0
$$




$$
\begin{aligned}
& \frac{\partial p}{\partial t}-c^{2} \frac{\partial \rho}{\partial t}+u\left(\frac{\partial p}{\partial x}-c^{2} \frac{\partial \rho}{\partial x}\right)=0 \\
& \frac{\partial p}{\partial t}+\rho c \frac{\partial u}{\partial t}+(u+c)\left(\frac{\partial p}{\partial x}+\rho c \frac{\partial u}{\partial x}\right)+\frac{\rho u c^{2}}{A} \frac{\partial A}{\partial x}=0
\end{aligned}
$$

Where $c=\sqrt{\gamma p / \rho}$ is the local speed of sound. The considered nozzle problem has two different cases for the outflow boundary conditions. One, is supersonic outflow boundary conditions and the other is subsonic.

\section{1- Supersonic Outflow Boundary Conditions}

The flow at the nozzle exit is supersonic, this means that all of the waves are forced to move out of the computational domain. Therefore, the compatibility and the original governing equations are identical. There is a need for some special boundary treatment. The spatial derivatives of all the governing equations are discretized with backward differences.

\section{2-Subsonic Outflow Boundary Conditions}

When the pressure at the nozzle exit increases compared to the total inlet pressure, a shock is formed in the divergent part of the nozzle. In this case, the outflow at the nozzle exit is subsonic with the acoustic perturbations. Based on the analysis of the characteristic theory, there are two outgoing characteristic waves and one incoming characteristic wave. The outgoing waves are the acoustic wave with the velocity $(u+c)$ and the entropy wave of the velocity $u$ (the entropy wave formed inside the nozzle). The incoming wave is the acoustic wave with the velocity $(u-c)$. According to the idea of non-reflecting boundary conditions of Thomson, we must suppress the incoming acoustic wave. To do so we consider the compatibility equations $R 2$ and $R 3$ and instead of the compatibility equations $R 1$ we use the following equation:

$$
\frac{\partial p}{\partial t}-\rho c \frac{\partial u}{\partial t}=0
$$

\section{NUMERICAL RESULTS}

Simulating acoustic-shock waves interaction in quasi-one dimensional convergingdiverging nozzle is performed by using fourth-order, 3-point stencil, compact McCormick-type scheme for spatial derivative approximations and the optimized 5-6, 2-N storage Low-Dispersion and-Dissipation Runge-Kutta method (LDDRK) as a time marching scheme.

According to Fourier analysis of the considered compact scheme, the numerical wave number is purely real. This is a property of central schemes. This means that, the considered compact scheme has a little own dissipation. Therefore, simulating nonlinear problems require additional terms to damp the non-physical waves. In the discretization of the conservative governing equations we use explicit damping terms in order to suppress the spurious waves that generated during the computations. 
A steady-state solution is obtained by implementing the mentioned numerical schemes until residuals are driven to machine zero. The steady state computation requires approximately 5000 times the time increment $\Delta t$. After the steady state is achieved we consider the incidence of the acoustic wave defined by the equation (11) on the inlet of the nozzle $(x=-200)$, then we perform the problem for two different cases. One is without shock and the other is where a normal shock is formed in the divergent section of the nozzle. In the first case, we investigate the numerical acoustic solution. As soon we can resolve the acoustic waves we turn to investigate the effect of the normal shock on the acoustic waves. For our computations, we use the time step $\Delta t=0.3$ with spatial grid spacing $\Delta x=0.5$ in both cases.

For the first case, the inflow boundary conditions are subsonic (Mach number equal to 0.5 ). For boundary conditions treatment, we use radiation boundary conditions for the subsonic inflow boundary conditions. The outflow boundary conditions are supersonic (Mach number equals 1.55 ) so, there is no need for special treatments. We use 3-points stencil damping method for the interior points where no damping used for the terminal points $(x=-200 \& x=80)$ of the computational domain. The damping coefficient is a free parameter used to control the damping terms. By numerical experiments, the small damping can not suppress the spurious waves and on the other hand higher damping values have harmful effects on the acoustic waves. Therefore, we can say that, the damping coefficient plays the key role in the success of the computation. In our computation we use the value 0.9 for the damping coefficient. The computed pressure fluctuations are shown in figure (7). From the presented results, it is clear that, no significant effect for the numerical dissipation on the acoustic solution.

In the second case, a normal shock is formed in the nozzle by increasing the exit pressure with respect to the total inlet pressure. The inflow boundary conditions are subsonic (Mach number equals 0.5). As in the first case, we use the radiation boundary conditions for boundary condition treatment. The outflow boundary conditions are subsonic (Mach number equals to 0.6 ). We employ the characteristic boundary conditions. According to the presence of the normal shock we use a pressure sensor $v_{i}=\left|p_{i+1}-2 p_{i}+p_{i-1}\right| /\left|p_{i+1}+2 p_{i}+p_{i-1}\right|$ to locate where the greatest pressure gradient is and then choose the value of the damping coefficient consequently. We used the 3-points stencil damping method interior points of the computational domain where no damping is used for the terminal points $(x=-200 \& x=80)$. In the computation of this case we use the value 0.6 for the damping coefficient and increase it to 2.4 around the greatest pressure gradient where the shock located. The computed acoustic solution is shown in figure (8), this figure shows one snapshot for spatial distribution of pressure fluctuations in the presence of a normal shock. From the presented results, it is clear that, the interaction between shock wave and acoustic wave amplify pressure fluctuations. 
Figure (9) shows the numerical mean flow pressure in the presence of shock wave. The numerical solution shows very small and a non-significant wiggles around the shock. In figure (10) we see a very good agreement between the exact mean flow pressure (represents by line) and the numerical mean flow pressure (represents by bold black circles).

\section{9- CONCLUSION}

From the previous discussion we conclude that, the fourth-order compact McCormick-type scheme with the 5-6, 2-N storage Low-Dispersion and-Dissipation Runge-Kutta combined with a carefully selected damping coefficients perform the considered problem very well. According to small stencil, direct computations without matrix inversions and the capability to resolve waves with a high wave number limit, the indicated compact scheme is strongly recommended in acoustics computations.

\section{0- REFERENCES}

1- Lele, S. K. "Compact Finite Difference Schemes with Spectral-Like Resolution" Journal of Computational Physics Vol. 103, pp. 16-42, (1992)

2- Sheg-Tao Hu, Kwang-Chung Hsieh, and Y.-L. Peter Tsai "Simulating Waves in Flows by Runge-Kutta and Compact Finite Difference Schemes "AIÂA Journal,

3- Hixon, R., "A New Class of Optimized High-Accuracy Compact Schemes" AIAA paper 98-0367, (1998).

4- Hixon, R. and Turkel, E. "Compact Implicit MacCormack-Type Schemes with HighAccuracy" Journal of Computational Physics, Vol. 158, pp. 51-70, (2000).

5- Hu, F. Q. Hussaini, M. Y and Manthey, J. LLow-Dissipation and LowDispersion Runge-Kutta Schemes for computational Acoustics "Journal of Computational Physics, Vol.124, pp. 177-191, (1996).

6- Stanescu, D and Habashi, W. G." 2N-Storage Low-Dissipation and LowDispersion Runge-Kutta Schemes for Computational Acoustics" Journal of Computational Physics Vol. 143, pp. 674-681, (1998).

7- Tam, C. K. W., and Shen, H, "Direct Computation of Nonlinear Acoustic Pulses using High Order Finite Difference Schemes", AIAA Paper 93-4325, (1993).

8- Vichnevetsky, R. and Bowels, J. B., "Fourier Analysis of Numerical Approximations of Hyperbolic Equations " (SIAM Philadelphia), (1982).

9- Khalid M. Hosny, "Erratum", Journal of Computational Physics Vol. 163, pp. 547. (2000).

10- Hardin, J. C., Ristorcelli, J. R. and Tam, C. K. W. (Editors), "First ICASE/LaRC Workshop On Benchmark Problems in Computational Aeroacoustics (Hampton, VA), NASA CP. 3300, (1995)

11- Bui, T. T. and Mankbadi, R. R., "Direct Numerical Simulation of Acoustic Waves Interacting With A Shock Wave In A Quasi-1D Convergent-Divergent Nozzle Using Unstructured Finite Volume Algorithm " International Journal of Computational Fluid Dynamics, Vol. 10, pp. 281-298, (1998).

12- Tam, C. K. W. and Zhong Dong, "Radiation and Outflow Boundary Conditions for Direct Computation of Acoustic and Flow Disturbances in a Non-uniform Mean Flow" Journal of Computational Acoustics Vol. 4, No.2, pp. 175-201, (1996).

13- Thompson, K. W., "Time-Dependent Boundary Conditions for Hyperbolic System", Journal of Computational Physics Vol. 68, pp. 1-24, (1987). 


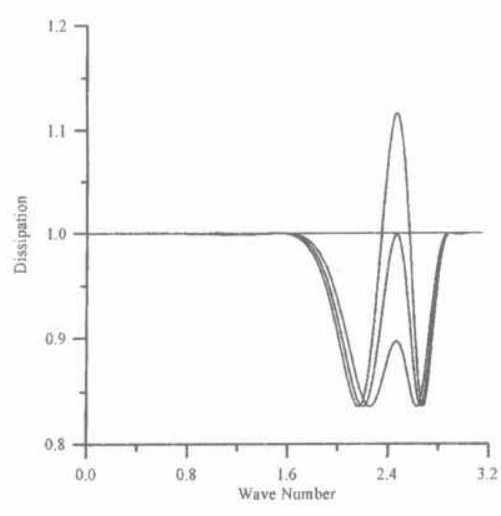

Fig. (1): Numerical Dissipation

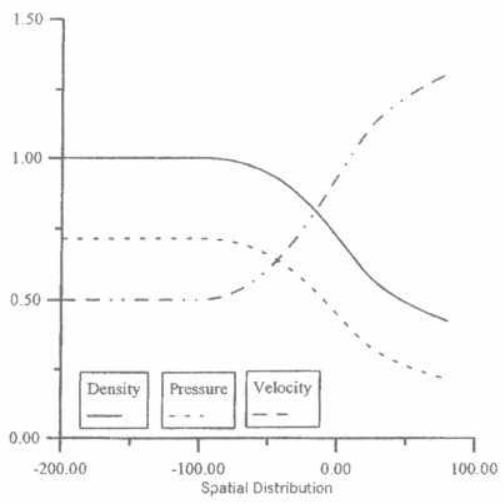

Fig. (3): Initial Steady-State

Solutions

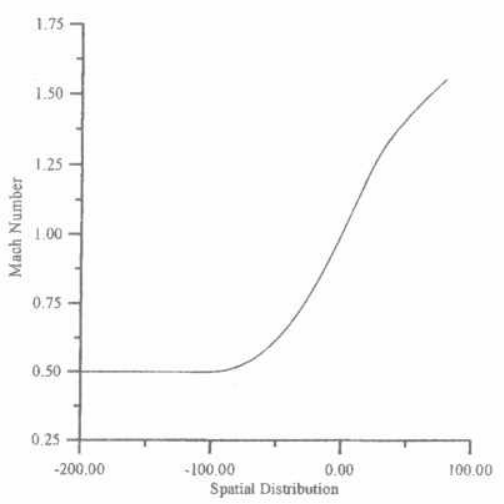

Fig. (5): Mach Number (Without shock)

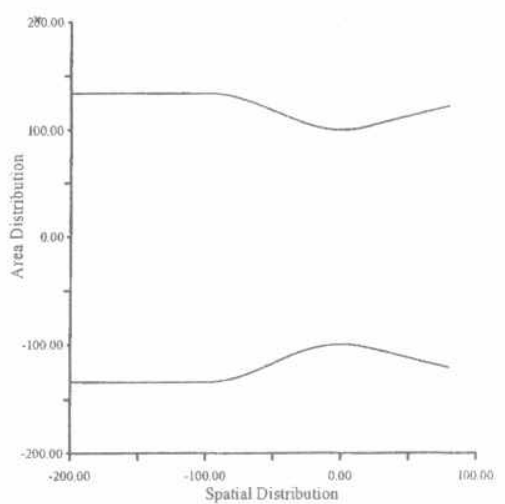

Fig. (2): Nozzle Geometry

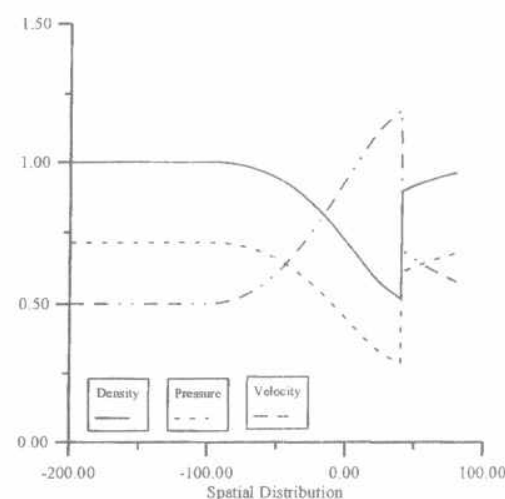

Fig. (4): Initial Steady-State

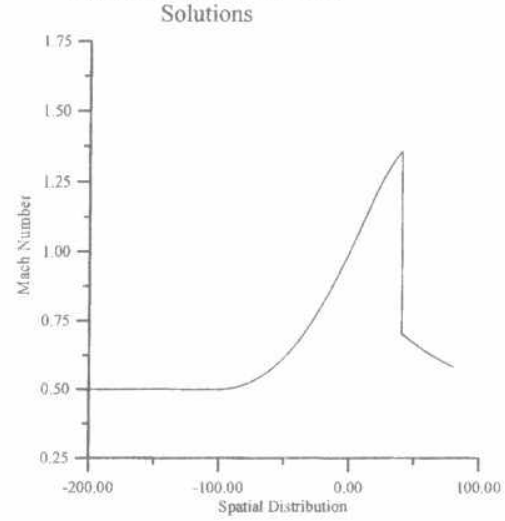

Fig. (6): Mach Number (With shock) 


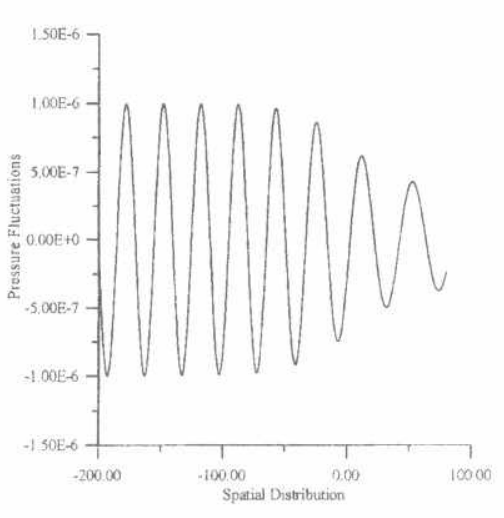

Fig. (7): One Snapshot of Pressure Fluctuations (Without shock)

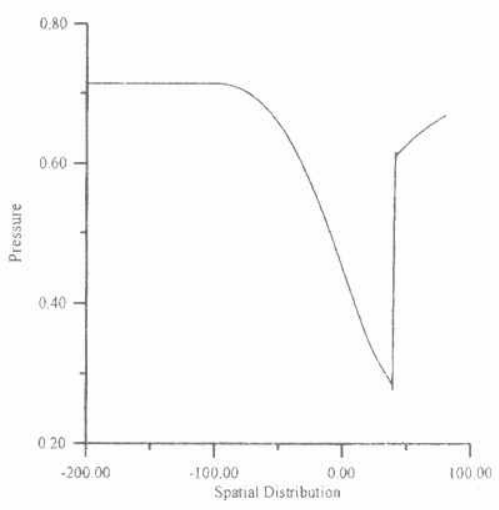

Fig. (9): Numerical Mean Pressure (With shock)

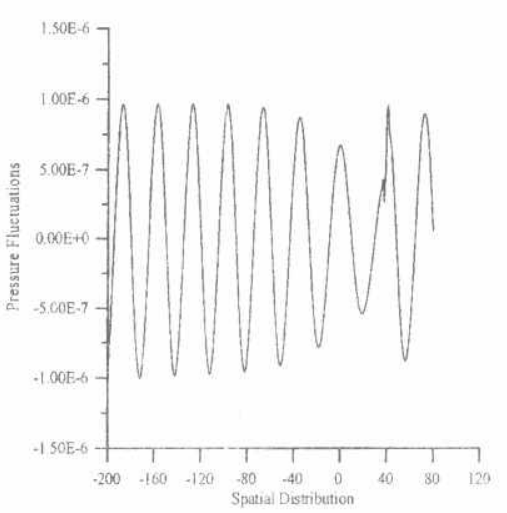

Fig. (8): One Snapshot of Pressure Fluctuations (With shock)

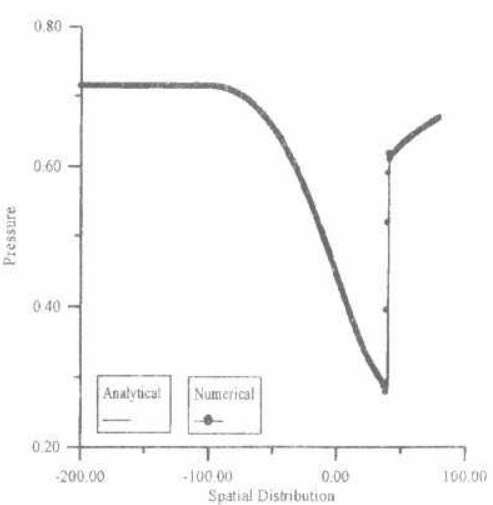

Fig. (10): Numerical \& Analytical Mean Pressure (With shock) 